\title{
Acerca de la enseñanza de la lengua oral
}

\author{
Montserrat Vila e Ignasi Vila
}
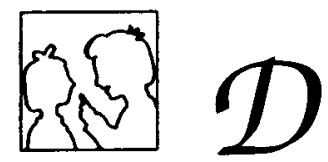

La enseñanza de la lengua oral es tan importante como la enseñanza de la lengua escrita. Ambos procesos de enseñanzalaprendizaje son objetivos de etapa y no de área, es decir que durante todas las etapas de educación obligatoria (infantil, primaria y secundaria), los objetivos referidos al lenguaje, ya sea oral o escrito, requieren una metodología compleja, una minuciosa planificación, y evidentemente una evaluación, que si bien deben priorizarse en el área de lengua, conciernen a todas las áreas del curriculum. Los autores nos presentan un conjunto de criterios para la programación de actividades de enseñanzalaprendizaje de la lengua.

\section{INTRODUCCION}

La actual reforma educativa que se sigue en el Estado Español rompe una lanza en favor de la lengua oral. Tradicionalmente, la lengua oral había sido vista como subordinada a la lengua escrita, de modo que era ésta última la que acaparaba el máximo de atención por parte de los docentes. Hoy en día, las cosas están cambiando. Por ejemplo, en relación con los distintos objetivos que los nuevos Diseños Curriculares proponen para la etapa de la Educación Secundaria Obligatoria está el tratamiento de forma semejante de la lengua oral y la lengua escrita y, por tanto, se afirma que los escolares, al finalizar la etapa, deben ser capaces de «comprender y producir mensajes orales y escritos con propiedad, autonomía y creatividad (...) usándolos para comunicarse y para organizar los propios pensamientos y reflexionar sobre los procesos implicados en el uso del lenguaje». El ejemplo sirve para mostrar que en los actuales planteamientos educativos se anima tanto la enseñanza de la lengua oral como de la lengua escrita y, además, estas ideas alcanzan a todas las etapas de la educación obligatoria: infantil, primaria y secundaria.

Este planteamiento tiene numerosas implicaciones, aunque en relación con este artículo nos interesa destacar dos. Primero, los objetivos referidos a las capacidades que los escolares deben desarrollar en relación con el lenguaje son objetivos de etapa y no de área. En este sentido, su desarrollo está en relación con el conjunto de actividades de enseñanza-aprendizaje que las alumnas y los alumnos realizan y no únicamente con las actividades que se derivan del área de lengua. Segundo, las capacidades que los escolares deben desarrollar en relación con el uso de la lengua oral no se limitan a su propiedad comunicativa en los distintos contextos de uso, sino que 
van más lejos y la oralidad se encara como reguladora de la propia conducta y como herramienta para reflexionar sobre sus propios mecanismos de uso y, por tanto, como instrumento de aprendizaje del uso de la misma lengua oral. Además, se asume que el aprendizaje y la transformación de conocimientos está en relación con el dominio de la lengua.

No obstante, un gran número de profesoras y profesores se muestran escépticos respecto a dicha enseñanza. De una parte, entre amplios sectores del profesorado se mantiene la creencia de la primacía de la lengua escrita sobre la lengua oral. De la otra, los objetivos concretos que se persiguen a través de dicha enseñanza y los contenidos específicos a utilizar para conseguirlos están poco definidos, lo cual se agrava cuando se quieren secuenciar. Además, las actividades de lengua oral requieren una metodología difícil y compleja, una minuciosa planificación, una realización a veces imprevisible, una determinada actitud por parte de profesorado y alumnado y, por último, en el ámbito de la evaluación, las dificultades son enormes tanto desde el punto de vista de las formas que debe adoptar como de los contenidos a evaluar. Probablemente, este segundo aspecto es el más preocupante, ya que cuando existe un sector del profesorado que se decide a trabajar la lengua oral encuentra tantas dificultades que, en muchos casos, abandona el empeño.

En este artículo exponemos algunas de las características actuales de la enseñanza de la oralidad a partir de un trabajo realizado con profesoras y profesores de la Enseñanza Secundaria de Catalunya que realizan actividades de enseñanza-aprendizaje de la lengua oral (Vilà y Vila, 1992a) a través del cual ejemplificamos los problemas reales con que se enfrenta el profesorado cuando se decide a incorporar a su práctica profesional dicha enseñanza. La segunda parte del artículo reflexiona sobre el concepto de oralidad y sugiere un conjunto de criterios que, a nuestro entender, sirven para organizar secuencias didácticas dirigidas a la enseñanza de la lengua oral.

\section{LA LENGUA ORAL EN LA ENSEÑANZA SECUNDARIA DE CATALUÑA}

Los estudios que muestran la falta de intencionalidad educativa en el ámbito de la lengua oral comienzan a ser numerosos. Así, Bassedas y Vila (1993), en un trabajo realizado a partir de una muestra de escuelas infantiles y parvularios de un barrio de la ciudad de Barcelona, evidencian que en el primer ciclo de la Educación Infantil, el $61 \%$ de las actividades lingüísticas que realizan las maestras con una clara intencionalidad educativa se relacionan con la comprensión y expresión oral y, sin embargo, en el segundo ciclo de la Educación Infantil dicho porcentaje se reduce a un $24 \%$. Igualmente, Vilà y Vila (1992b), tras realizar un estudio con el conjunto de alumnas y alumnos de primer curso del conjunto de los estudios de Magisterio de Cataluña, constatan que la percepción subjetiva que tienen estos jóvenes sobre su participación en actividades de enseñanza-aprendizaje de la lengua oral a lo largo de la Enseñanza Secundaria es muy pequeña. Por ejemplo, el $50 \%$ afirma no haber realizado nunca a lo largo de los cuatro cursos exposiciones orales en lengua catalana y el $35 \%$ afirma no haberlo hecho en lengua castellana. Estos porcentajes aumentan ostensiblemente en relación a actividades como debates, mesas redondas, dramatizaciones o semejantes. De hecho, sólo aceptan haber realizado actividades de lectura expresiva.

A nuestro entender, como ya hemos dicho, existen dos razones fundamentales para comprender la falta de presencia de actividades intencionalmente educativas referidas a la enseñanza de la lengua oral a lo largo de la enseñanza obligatoria. En primer lugar, la influencia sobre el profesorado de una tradición lingüística que prioriza en sus análisis y descripciones la lengua escrita sobre la lengua oral. De hecho, 
muchos profesores piensan de forma más o menos consciente que la lengua oral «crece» y se «desarrolla» de forma natural sin que sea necesario enseñarla de forma sistemática, a diferencia de la lengua escrita que requiere un trabajo propiamente escolar. En segundo lugar, cuando la lengua oral se ha introducido en las aulas lo ha hecho de la mano de incomprensiones y excesos relacionados con el uso y abuso del término «competencia comunicativa» acuñado por Hymes (1971). De hecho, las creencias del profesorado sobre un supuesto «crecimiento» natural de la lengua oral se han mantenido y se ha justificado la falta de dominio de la lengua oral por parte de los escolares a través de la pobreza linguíística de sus relaciones sociales. Así, se ha defendido y aún se defiende con fuerza que las niñas y los niños de nuestros pueblos y ciudades están sometidos a las órdenes del adulto y a la tiranía del televisor con la consiguiente merma de auténticas situaciones comunicativas que permitan el desarrollo de capacidades relativas al uso de la lengua oral. Por eso, en muchos casos, se ha incorporado la lengua oral a las aulas para "compensar» la falta de situaciones comunicativas extraescolares y se ha hecho a partir de la idea de que «a hablar se aprende hablando». En consecuencia, en bastantes aulas, especialmente de la Enseñanza Primaria, se han introducido debates, asambleas, lecturas en voz alta, etc., para que los alumnos pudieran hablar y expresarse. Pero, estas prácticas se han realizado de forma espontánea sin que, en la mayoría de los casos, se ofreciera a los escolares los recursos y procedimientos apropiados para hablar y expresarse mejor y sin que se fomentaran de forma intencional las actitudes necesarias para mejorar las capacidades expresivas en el ámbito de lo individual. Además, en algunos casos, a las ideas expuestas se sumaba un supuesto quehacer relacionado con la creatividad. Este espontaneísmo que Camps (1990) extiende a las actividades lingüísticas en general ha servido bastante poco para mejorar las habilidades lingüísticas de los escolares con su consiguiente abandono de una parte del profesorado que se muestra escéptico ante los resultados y que prefiere ser consistente en su práctica educativa con sus propias creencias.

Ambas razones - creencias inadecuadas sobre el desarrollo de las capacidades relativas a la lengua oral y espontaneísmo por parte del profesorado que intenta trabajar la lengua oral - son, a nuestro entender, las causas fundamentales por las que la lengua oral está relegada en las programaciones del profesorado y por las que existen muy pocos recursos metodológicos para trabajarla de forma sistemática.

En el trabajo realizado por nosotros mismos con 405 profesoras y profesores de lengua de la Enseñanza Secundaria en Catalunya se corraboran estas apreciaciones. Así, un $40 \%$ que dice realizar exposiciones orales con sus alumnos reconoce que una parte de ellos o, incluso, la mitad siempre se queda al margen de esta actividad. De hecho, sólo un $48 \%$ dice que todos sus alumnos realizan exposiciones orales. Además, la mayoría de los que realizan exposiciones orales (un 75\%) dice que las exposiciones de sus alumnos se asemejan a una lectura en voz alta. Por último, la inmensa mayoría de los que realizan estas actividades las limitan a «sacar a la pizarra» a un alumno a contestar alguna pregunta o a exponer una parte de la lección que se le ha pedido que la aprenda en la clase anterior. De hecho, el profesorado reconoce que en estas actividades no existe intención educativa, compartida con sus alumnas y alumnos, referida al trabajo de la oralidad. Ciertamente, existe un pequeño grupo de profesoras y profesores que dice trabajar con grupos reducidos con el objeto de que expongan el trabajo realizado al conjunto de la clase, profesor y compañeros.

Otro aspecto de interés del trabajo realizado se relaciona con las actividades de evaluación del profesorado de la Enseñanza Secundaria. Así, la mayoría de las personas encuestadas afirman tener en cuenta la lengua oral en la evaluación. Sin embargo, un $80 \%$ confiesa que ello se limita a fijarse, a veces, en cómo hablan sus alumnos. Sólo un 4,55\% afirma realizar evaluaciones concretas de las acticvidades programadas para trabajar la oralidad. 


\section{8}

En definitiva, al menos en Cataluña, la enseñanza de la lengua oral en la Educación Secundaria es colateral y no forma parte del núcleo de las programaciones del profesorado. Ciertamente, existe un pequeño grupo de profesoras y profesores que se preocupan por el tema y que se esfuerzan por desarrollar metodologías adecuadas para mejorar la lengua oral de sus alumnos. En lo que sigue ofrecemos nuestro punto de vista sobre la forma de abordar la oralidad a lo largo de la Enseñanza Primaria y Secundaria. No cabe decir que una parte importante de nuestras reflexiones se sustentan en la discusión de nuestra propia práctica educativa y de otras profesoras y profesores que, como nosotros, están interesados en el desarrollo de experiencias y materiales que permitan tratar de forma sistemática e intencional el desarrollo de capacidades relativas al uso de la lengua oral de los alumnos a lo largo de la enseñanza obligatoria.

\section{ALGUNAS CUESTIONES ACERCA DE LA ENSEÑANZA DE LA LENGUA ORAL}

Generalmente, existe una tendencia a separar la lengua oral y la lengua escrita. Así, se enfatizan las diferencias estructurales y funcionales entre ambos códigos y se postulan criterios diferentes para abordar su tratamiento educativo. Sin embargo, desde una perspectiva psicolingüística (Scinto, 1986), es difícil de mantener una separación tajante entre ambos códigos y, por el contrario, se postulan relaciones de horizontalidad de modo que, en el proceso de enseñanza-aprendizaje de la lengua, tanto la lengua oral como la lengua escrita deben tener un tratamiento equilibrado y complementario. Frecuentemente, los usos orales y escritos de la lengua se interrelacionan y se crean situaciones de comunicación mixtas en las que participan ambos códigos (Gregory y Carroll, 1978; Marí, 1982; Cassany, 1989; Vilà, 1991; Vilà y Ribas, 1993).

Desde una perspectiva comunicativa, el uso adecuado del lenguaje supone saber concretar, en un contexto determinado, la opción más eficaz del conjunto de potencialidades que ofrece una lengua concreta (Payrató, 1988). En este sentido, hablar o escribir significa escoger y el hablante/escritor escoge aquello que cree más adecuado en el contexto en que se encuentra para que se reconozcan sus intenciones, lógicamente en función de la capacidad y la habilidad con que pueda hacerlo. Metodológicamente, ello significa, en relación a la lengua oral, que en las aulas se deben crear situaciones reales de comunicación en las que los escolares tengan que utilizar la lengua para transmitir sus intenciones a unos interlocutores determinados y en una situación concreta. La claridad, precisión y eficacia de las comunicaciones orales de los escolares creemos que está en relación con la posibilidad de poder reflexionar sobre el contenido y la forma lingüística de sus intervenciones antes de ser emitidas. En otras palabras, pensamos que debe existir un equilibrio entre el uso de la lengua oral y la reflexión metacomunicativa y metalinguística como forma de incidir en la mejora de las producciones orales.

En segundo lugar, la comunicación es un proceso bilateral entre interlocutores, incluso en los usos monológicos, y no es un proceso unilateral entre un emisor y unos receptores. Así, la comunicación es un proceso interactivo y, por tanto, es esencial determinar las operaciones lingüísticas y cognitivas que deben realizar cada uno de los interlocutores, tanto quien emite un mensaje como quien lo escucha o lo lee. Desde el punto de vista de los receptores, este punto de vista es básico si pretendemos asegurar, en el ámbito de la lengua oral, una actitud de escucha activa y comprensiva que garantice la comunicación. Al hacerlo de este modo, las actividades de lengua oral que se realizan en el aula no sólo son rentables para los escolares que producen mensajes orales sino para el conjunto de alumnas y alumnos. Además, esta 
forma de trabajo posibilita que el locutor obtenga una respuesta inmediata a partir de las reacciones - verbales y no-verbales - de sus interlocutores, de modo que pueda modificar su discurso oral de la manera que considere más conveniente para aumentar su eficacia comunicativa. Justamente, como hemos señalado anteriormente, una de las habilidades más importantes que deben adquirir los hablantes consiste en la capacidad de modificar su discurso en función de las circunstancias contextuales en que éste se produce. Por eso, si tenemos en cuenta simultáneamente producción y escucha, la fase de planificación del texto oral debe incluir, además de otras operaciones, la previsión de diversos itinerarios orales en función del contenido a transmitir y el uso de diferentes recursos oratorios para garantizar una comunicación eficaz.

En tercer lugar, la comunicación oral es un proceso irreversible, de modo que los interlocutores deben grabar y memorizar lo que van diciendo y escuchando en un tiempo determinado. Ello implica dos aspectos. De una parte, una rápida selección y organización de las ideas y, de la otra, la imposibilidad de hechar marcha atrás y borrar lo que ya está dicho u oído. Desde el punto de vista de la producción, las pausas, las vacilaciones o las repeticiones múltiples en el discurso de un locutor son claros ejemplos de las dificultades que comporta la comunicación oral. Por eso, como ya hemos dicho, la fase de planificación o de preparación previa a la exposición oral es esencial en el proceso de composición de textos orales complejos.

Desde una perspectiva lingüística, la lengua oral que debe ser objeto de enseñanza-aprendizaje en el contexto escolar no se refiere exclusivamente a sus usos informales. Por el contrario, garantizados ciertos niveles de fluidez y expresión en contextos coloquiales, la enseñanza-aprendizaje de la lengua oral se acerca notablemente a algunas de las cualidades hasta ahora limitadas al texto escrito. Así, los usos formales de la lengua oral requieren un nivel de contextualización, realizado a través de marcadores del discurso, similares al texto escrito (Vila, 1993). Así, la capacidad de producir textos orales complejos requiere el dominio del aparato formal lingüístico que permite la coherencia y la cohesión del discurso. No nos extendemos en este punto porque varios artículos de este monográfico se refieren a él.

En definitiva, proponemos un tratamiento de la lengua oral en el contexto escolar que comparte características comunes y complementarias con el tratamiento de la lengua escrita. La adquisición de la competencia comunicativa oral se garantiza, desde nuestro punto de vista, desde la potenciación de los distintos usos de la lengua y, especialmente, en la enseñanza-aprendizaje de los usos monológicos en contextos de formalidad media-alta y desde la reflexión previa y posterior al uso de la lengua. En este sentido, es tan importante posibilitar contextos reales de uso de la lengua oral como potenciar el acceso a lecturas y a la recopilación escrita de informaciones que permitan seleccionar, completar y ordenar el conocimiento de los escolares sobre el tema que debe ser objeto de la expresión oral. Creemos que actividades como consultar bibliografía, preparar un guión o un esquema de apoyo previo a la exposición oral, escuchar la grabación de un texto previamente expuesto, etc no sólo facilita la participación en actividades de lengua oral (da seguridad, permite que no intervengan siempre los mismos escolares, etc) sino que ayuda a mejorar la forma y el contenido del discurso oral.

\section{ALGUNOS CRITERIOS PARA LA PROGRAMACION DE ACTIVIDADES DE ENSEÑANZA-APRENDIZAJE DE LENGUA ORAL}

La pretensión de desarrollar un enfoque comunicativo en la enseñanza-aprendizaje de la lengua obliga a definir sus características básicas ya que bajo la etiqueta "comunicativo-funcional" se esconden propuestas muy diversas e incluso contradictorias (Nunan, 1989; Vila, 1989). El enfoque que proponemos consta de cuatro 
aspectos. En primer lugar, centra su interés en mejorar la eficacia comunicativa de las alumnas y los alumnos. En segundo lugar, prioriza la enseñanza de los procedimientos implicados en la composición de los textos orales y considera que la intervención pedagógica debe realizarse a lo largo de dicho proceso. En tercer lugar, integra el conocimiento del aparato formal lingüístico y el dominio instrumental del lenguaje y, por último, adopta una perspectiva cognitiva.

Desde esta perspectiva, una programación y, a la vez, evaluación de la lengua oral debería contemplar la correción o incorrección del sistema de la lengua utilizada, la capacidad para utilizar los mecanismos de adecuación a la situación y al contexto comunicativo, el uso de los elementos que rigen la coherencia y cohesión de los diferentes textos orales, el dominio en la regulación de la interacción y el uso adecuado de elementos extralingüísticos como la entonación, el ritmo, la gesticulación, etc. $y$, finalmente, el conocimiento del tema que es objeto del texto oral en el grado de profundidad que se considere necesario. A la vez, una programación de lengua oral creemos que debe posibilitar a los escolares que construyan conocimiento sobre la utilidad de un uso adecuado de la lengua oral en su vida académica y social. Igualmente, debe permitir a las alumnas y a los alumnos que adquieran criterios para valorar el grado de adecuación/inadecuación de sus textos orales y, por último, debe promover actividades en las que los escolares tengan la oportunidad de emplear públicamente la lengua oral.

A modo de ejemplo a continuación presentamos de forma esquemática una secuencia didáctica relativa al proceso de composición de un texto oral monológico en el aula. En ella se especifica la función del locutor y, paralelamente, la función de los destinatarios del discurso oral, así como la función del profesor o de la profesora a lo largo del proceso. Tal y como hemos dicho, en la secuencia didáctica se establece un equilibrio entre el uso de la lengua y la reflexión metacomunicativa y metalingüística.

\section{Fase de planificación}

En todas las actividades está previsto un tiempo para preparar el texto sin perder de vista el propósito del discurso oral (informar, convencer, demostrar, etc.), el contenido a transmitir y el contexto comunicativo. En esta fase se trata de generar, seleccionar y ordenar información, consultar fuentes diversas, elaborar esquemas, mapas conceptuales, etc. para, de este modo, conseguir estructurar los ejes centrales de un discurso acorde con el objetivo propuesto. En esta fase, el tratamiento de la lengua oral tiene como referente la lengua escrita.

Durante la fase de planificación se preveen los distintos itinerarios para conducir el tema y, así, poder tener en cuenta los conocimientos previos de los destinatarios. Igualmente, se seleccionan los recursos de oratoria para mantener el interés de la audiencia y se preparan los soportes gráficos y ayudas visuales que permiten focalizar la atención del auditorio e ilustrar los contenidos del tema que se va a exponer.

En esta fase se promueve por parte del profesorado la reflexión sobre los elementos lingüísticos y comunicativos que intervienen en el discurso y siempre conlleva el uso de ensayos en privado para garantizar el éxito posterior.

\section{Fase de producción}

Desde el punto de vista del locutor, esta fase consiste en la emisión de un texto adecuado a las circunstancias contextuales. La puesta en escena o textualización del texto planificado comporta el dominio de los mecanismos de coherencia textual y la habilidad para detectar el grado de comprensión e interés de la audiencia. Igualmente, esta fase comporta el uso de las reglas lingüísticas y discursivas, así como el uso apropiado de los recursos extralingüísticos y de retórica para mantener el interés del auditorio. 
FIGURA 1

Proceso de composición de un texto oral monológico en un contexto escolar

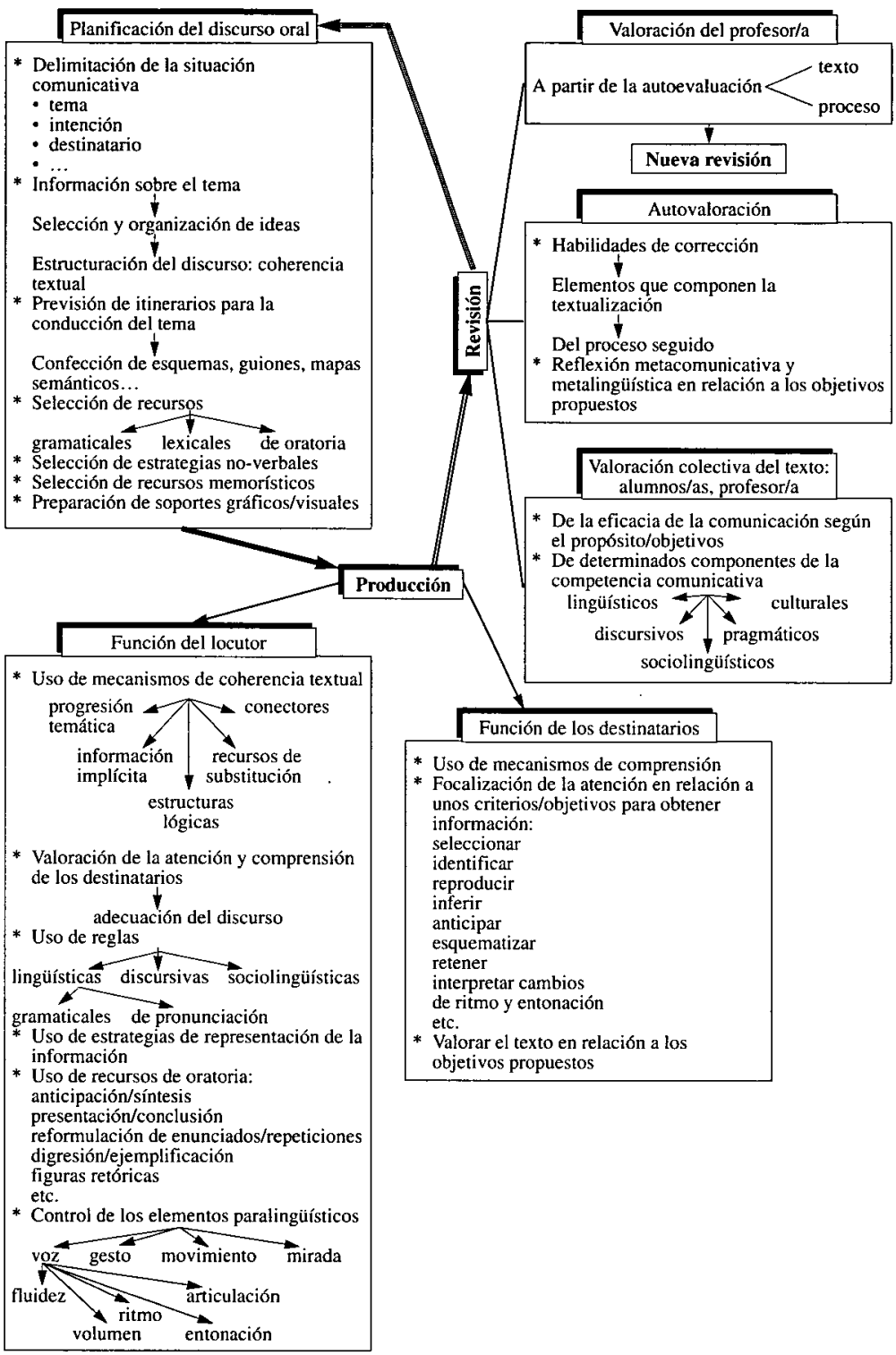

En esta fase se utilizan medios audiovisuales para la grabación de las intervenciones y su análisis posterior.

Desde el punto de vista de los destinatarios, en esta fase se promueve la recepción atenta y comprensiva del texto. La focalización de la arención de los escolares se debe realizar de acuerdo con unos criterios que previamente se hayan trabajado como son la selección, la identificación, la reproducción y la esquematización de informaciones, la capacidad de inferir nuevas informaciones o de relacionarlas entre sí, la contraargumentación, la interpretación de cambios de ritmo y entonación, etc. 
Fase de revisión

En primer lugar, sugerimos una valoración colectiva del texto oral expuesto en la que participen los escolares y la profesora. El grupo comenta las intervenciones orales en relación con los objetivos propuestos y se analiza el discurso a partir del uso realizado de los elementos lingüísticos, discursivos, sociolingüísticos, estratégicos y extralingüísticos, así como del contenido expuesto.

En segundo lugar, en esta fase fomentamos la habilidad de autocorreción y sugerimos al escolar que, de forma individual, reflexione sobre el discurso producido y lo revise con el objeto de elaborar una segunda versión mejorada. La autoevaluación presupone que el escolar disponga de la grabación del texto expuesto y de unas pautas de observación facilitadas por la profesora.

Por último, proponemos una evaluación final por parte del profesor, el cual valora la nueva versión del texto y en caso de que aún se detecten problemas se puede sugerir al alumno la realización de ejercicios específicos o se puede recomendar que elabore una nueva versión completa del texto oral.

\section{CONCLUSIONES}

A lo largo del artículo hemos explicitado las bases de una propuesta concreta para trabajar la lengua oral en el contexto escolar. Para finalizar queremos, a modo de resumen, sintetizar las ideas principales.

En primer lugar, creemos que la enseñanza de la lengua oral debería tender hacia la priorización de actividades de enseñanza-aprendizaje de textos o discursos monológicos formales. De hecho, las formas más habituales de comunicación son situaciones orales plurigestionadas y, por tanto, distintas en algunos aspectos a nuestra propuesta. No está en nuestro ánimo negar la enseñanza de las destrezas sociales presentes en los textos plurigestionados. Muy al contrario, los textos orales plurigestionados deberían tener una mayor presencia en la enseñanza desde los primeros niveles de escolarización. Sin embargo, creemos que estas habilidades, tal y como muestran numerosos trabajos, se incorporan desde contextos informales de intercambio social y, normalmente, las niñas y los niños entre 8 y 10 años son capaces de expresarse en forma fluída y eficaz en dichas situaciones. Tal y como señala Tusón (1991), de lo que carecen los escolares no es de la posibilidad de acceder a los registros informales de la lengua oral, sino de la posibilidad de acceder a sus registros formales. Así, a lo largo de la enseñanza obligatoria, las profesoras y los profesores piden en muy pocas ocasiones a sus alumnos que se expresen utilizando un registro formal que deba ser planificado con anterioridad y, de hecho, como hemos visto, muy pocos profesores se plantean la enseñanza de la lengua oral a partir de unos objetivos y unos contenidos concretos.

Una segunda idea que hemos defendido se relaciona con la posibilidad de crear situaciones reales de comunicación en el contexto del aula, de modo que los locutores tengan cosas que decir y el auditorio esté interesado en lo que se dice. Ciertamente, ello resulta a veces muy difícil y, en último término, se trata de encontrar temas de exposición que tengan sentido para los escolares en el contexto de enseñanza-aprendizaje que es la escuela. No se trata de inventar temas o de incorporar conversaciones que son habituales entre los escolares de 10 a 16 años, sino de aprovechar los temas relevantes que forman parte del propio contexto escolar. En este sentido, es difícil enseñar la lengua oral que proponemos al margen de los contenidos de las distintas áreas curriculares. 
En tercer lugar, pensamos que una parte esencial del proceso de enseñanzaaprendizaje de la lengua oral remite a las fases de planificación y de revisión. Se trata de practicar la máxima de que «a hablar se aprende hablando si alguien enseña a hablar mejor de como ya se habla». A veces, la enseñanza de la lengua oral enfatiza exclusivamente la fase de producción y asume la creencia que basta con hablar para hablar mejor. Evidentemente no compartimos este punto de vista y creemos que la mejora de la lengua oral de los escolares está muy relacionada con que construyan conocimiento sobre la necesidad de planificar el texto oral y, consecuentemente, aprendan las habilidades implicadas en las capacidades relativas al uso de la lengua oral.

En cuarto lugar, defendemos que la enseñanza de la lengua oral debe enfatizar tanto las habilidades productivas como las receptivas. Los aspectos relacionados con la focalización de la atención de los receptores normalmente no se tienen en cuenta y consideramos que son una parte tan importante como la propia exposición. Por eso, para hacer posible este objetivo, los escolares y el profesor deben compartir el contenido de las actividades propuestas, los objetivos que se persiguen y, en último término, los criterios de evaluación.

Por último, queremos resaltar que todo lo anterior ha de permitir el trabajo consciente e intencional del profesor sobre las operaciones cognitivas y pragmáticas que regulan la producción oral de un texto formal. Por eso, creemos que dicho trabajo tiene mayor garantía de éxito en las fases de planificación y revisión que en la de producción. Entre otras cosas, las actividades de enseñanza-aprendizaje de la lengua oral han de potenciar también la seguridad y la desinhibición de los escolares, lo cual se consigue ofreciendo recursos y ayuda en la fase de planificación y a través del tratamiento individual y discreto de la corrección.

\section{Referencias}

BASSEDAS, M. y VILA, I. (1993) ¿Qué escuela para los niños de tres años?, en Aula de Innovación Educativa, 11, pp. 9-12

CAMPS, A. (1990) «Los objetivos lingüísticos de la educación», in SiguAN, M. (Ed.) Lengua del alumno, lengua de la escuela, Barcelona, PPU

CASSANY, D. (1989) Descriure Escriure, Barcelona, Empúries

GREGORY, M. y CARROL, S. (1978) Language and Situation, Londres, Rouledge y Kegan Paul

HYMES, D. (1971) Competence and performance in linguistic theory, en HUSLEY, R. y INGRAM, D. (Eds.) Language Acquisition: Models and Methods, Londres, Academic Pres

Nunan, D. (1989) Designing Tasks for Communicative Classroom, Cambridge, Cambridge Language Teaching Library

PAYRATÓ, L. (1988) Català col.loquial. Aspectes d'ús corrent de la llengua catalana, Valencia, Universidad de Valencia

SCINTO, L. (1986) Written Language and Psycbological Development, Nueva York, Academic Press

Tusón, A. (1991) «Iguales ante la lengua, desiguales ante el uso. Bases sociolingüísticas para el desarrollo discursivo", en Signos, 2, pp. 50-59

VILA, I. (1989) «La metodología en la enseñanza de segundas lenguas y el enfoque comunicativo, in Comunicación, Lenguaje y Educación, 2, pp. 91-93

VILA, I. (1993) “Psicología y enseñanza de la lengua», en Infancia y Aprendizaje, 62-63, pp. 219-229

VILÀ, M. (1991) "La planificació i el tractament de l'expressió oral», en Comunicacions del Segon Simposi sobre Ensenyament del Català als No Catalanoparlants, Vic, EUMO

VILÀ, M. y RIBAS, T. (1993) «La lengua oral en la formación inicial del profesorado», en SIGUAN, M. (Ed.) Enseñanza en dos lenguas, Barcelona, Horsori/ICE

VILÀ, M. y VILA, I. (1992a) La enseñanza de la lengua oral en la Enseñanza Secundaria, Informe no publicado, Barcelona, Institut de Ciències de l'Educació de la Universitat de Barcelona.

VILÀ, M. y VILA, I. (1992b) La lengua oral en la Enseñanza Secundaria. Estudio empírico de las opiniones del alumnado, Informe no publicado, Barcelona, Institut de Ciències de l'Educació de la Universitat de Barcelona. 


\section{Acerca de la enseñanza de la lengua oral Montserrat Vila e Ignasi Vila \\ CL\&E, 1994, 23, pp. 45-54}

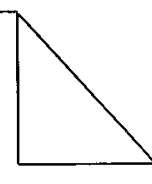

Resumen: El artículo señala la falta de experiencias y metodologías sobre la enseñanza de la lengua oral y ofrece varias explicaciones sobre ello. En la segunda parte se reflexiona sobre la necesidad de la enseñanza de la lengua oral y se propone un conjunto de criterios para la programación de actividades de enseñanza-aprendizaje.

Datos sobre los autores: Montserrat Vilá es profesora en la Universidad Autónoma de Barcelona, trabaja también en el departamento de Enseñanza de la Generalitat de Catalunya y en directora asociada de esta revista. Ignasi Vila es profesor en la Universidad de Gerona, trabaja también en el IMIPAE, del Ayuntamiento de Barcelona, y es director de CL\&E.

Dirección: Montserrat Vilà, Universitat Autònoma de Barcelona, Departament de Filologia Catalana, Edifici G, 08193 Bellaterra

Ignasi Vila, Universitat de Girona, Departament de Psicologia, Plaça Sant Domènec, 9, 17071 Girona.

(C) PERMISOS PARA CITAR O REPRODUCIR EN OTRAS FUENTES: Se pueden citar libremente hasta 500 palabras. Para reproducir una porción de texto mayor, figuras o ilustraciones, se deberá pedir permiso por escrito a la revista, especificando el uso al que se destina el texto. En todos los casos, se deberá citar el copyright de $C L \& E$. En el caso de artículos o textos que hayan sido a su vez reproducidos en $C L \& E$ los interesados deberán dirigirse tanto a los detentadores del copyright original como a $C L \& E$, en el caso de que se quiera hacer uso de la traducción. FOTOCOPIAS: Para todo lo relacionado con el uso mediante fotocopia del material de esta revista, deberán dirigirse a: CEDRO, C/ José Marañón, 10, 3. Izda. Tel. 5941575 . Fax 4453567 